\title{
Predictores académicos de desempeño en una Licenciatura en Música
}

Juan Pablo Correa y Rodolfo Raphael Moreno Martínez

\section{Resumen}

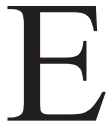
n este artículo se presenta una revisión de la literatura sobre predictores académicos de desempeño en el pregrado. Se enfatizan las variables evaluadas durante los procesos de admisión de programas de estudios musicales. A manera de conclusión, se proponen algunas preguntas que pueden guiar proyectos de investigación que exploren el potencial predictivo de variables, como el promedio de bachillerato, el puntaje del EXANI II, y las pruebas de admisión específicas al campo musical. Asimismo, se argumenta sobre la importancia de tener en cuenta las experiencias formativas preuniversitarias en el contexto mexicano, debido a las áreas de oportunidad que presenta la formación básica en música.

Palabras clave: predictores de desempeño, estudios musicales, pregrado, promedio de bachillerato, EXANI II, exámenes de admisión universitarios.

\section{Introducción}

Dos de los problemas más importantes a los que se enfrentan las instituciones de educación superior [IEs] en México son los bajos índices de eficiencia terminal y titulación. Esto se debe a diversas causas, tales como la deserción, reprobación, el bajo desempeño (De Vries, Arenas, Muñoz, y Saldaña, 2011), la aparente incapacidad de absorber la demanda creciente de educación superior (Chaín, Cruz, Martínez y Jácome, 2003) y los recursos limitados con los que cuentan las IES. Bajo estas condiciones, es importante seleccionar aspirantes con un perfil apropiado para cursar y egresar de los estudios superiores, en tiempo y forma. Un proceso de selección deficiente puede conllevar a índices altos de reprobación y deserción, así como a una inversión ineficiente de recursos públicos, debido a que los estudiantes con baja probabilidad de egreso llegan a consumir una cantidad importante de los recursos (Chaín et al., 2003).
En los programas de Licenciatura en Música, la eficiencia de este proceso de selección cobra mayor relevancia, ya que se encuentran entre los más onerosos del catálogo de carreras ofrecidas por las IES; esto a causa de que materias como Instrumento principal y Piano complementario son impartidas de manera individual; y otras como Música de cámara son impartidas en grupos pequeños -ejemplo, entre dos y cinco estudiantes (Wolf y Kopiez, 2014)-.

A esta problemática se añade el hecho de que el sistema nacional escolarizado no provee a los estudiantes de secundaria y bachillerato los conocimientos y habilidades óptimos para aspirar competitivamente a estudios musicales a nivel superior. Éste representa un reto para el diseño curricular, pues se deben encontrar estrategias para formar músicos profesionales a partir de un estado inicial de habilidades y conocimientos no idóneos con el fin de alcanzar, en cuatro o cinco años, los perfiles de egreso que exige el medio profesional nacional e internacional. El examen de admisión debe ser coherente con los perfiles del currículo, y debe ayudar a detectar a los candidatos más aptos para cursar y finalizar el currículo en tiempo y forma.

Por esta razón, es imperativo realizar estudios que permitan discernir y ponderar entre factores que conduzcan a una selección eficiente de candidatos a licenciaturas en música. En este artículo se presenta una síntesis de la revisión de la literatura que podría enmarcar este tipo de estudios, al mismo tiempo que se proponen algunas preguntas relevantes para el caso de la Licenciatura en Música de la Universidad Autónoma de Aguascalientes. Éste es el inicio de un proyecto de investigación, cuyo objetivo es explorar de qué manera el examen de admisión de conocimientos y habilidades musicales, junto con el EXANI II y el promedio de bachillerato, predicen el desempeño académico de los estudiantes del programa de licenciatura mencionado. El examen de admisión, el EXANI II y el promedio son las tres variables que se toman en cuenta para el proceso de admisión de los candidatos a la Licenciatura en Música de la UAA. El examen de conocimientos y habilidades musicales, especialmente diseñado y aplicado por el 
Departamento de Música de la referida institución, tiene una ponderación de $70 \%$, y está compuesto por una prueba teórica (35\%) y una audición con el instrumento (35\%). Tanto el EXANI II como el promedio de bachillerato equivalen, cada uno, a $15 \%$ de la evaluación. Se espera que los resultados obtenidos nos permitan comprender mejor la relación entre las variables mencionadas, en cuanto a su función y ponderación, y evaluar la eficiencia de este proceso de admisión.

\section{Predictores de desempeño académico a nivel de pregrado}

Las variables predictivas del desempeño académico universitario se pueden clasificar en tres grupos generales: $i$ ) personales, como la percepción de autoeficacia, motivación, bienestar psicológico, entre otras; ii) sociales, como nivel socioeconómico, etnia, religión, entre otras; iii) académicas, como los puntajes obtenidos en pruebas de admisión y el desempeño escolar previo (Albert, 2006; Arias, Chávez y Muñoz, 2006; Garbanzo, 2007). Debido a que la utilidad principal del proyecto de investigación que enmarca el presente artículo es proveer información relevante para evaluar un proceso de admisión a una Licenciatura en Música, nos enfocaremos en las variables de tipo académico.

En México, existe diversa literatura que correlaciona los puntajes obtenidos en los exámenes de admisión y el desempeño académico durante la carrera. Las variables académicas que se relacionan de manera directa con el desempeño son los exámenes requeridos para la admisión a las diversas IES -incluidos el EXANI II y otros particulares propios de cada institución-y el promedio de bachillerato. Se ha podido establecer una relación directa entre las áreas de razonamiento verbal, numérico y español del EXANI II con el rendimiento académico; además de que el resultado global de dicho examen permite calcular la probabilidad de buen desempeño académico del examinado (Chaín et al., 2003; Cortés y Palomar, 2008). En cuanto al promedio de bachillerato, diversos autores coinciden en señalar que el desempeño académico preuniversitario es uno de los mejores predictores de buen desempeño a nivel licenciatura (Escudero, 1984; González y López, 1985; Touron, 1985), pues éste no sólo resulta del dominio de los conocimientos y habilidades propios de los currículos de bachillerato, sino que también refleja aptitudes y hábitos de estudio puestos a prueba durante periodos de tiempo relativamente largos.

El EXANI II y el promedio de bachillerato se "orientan en torno al dominio de contenidos y habilidades defini- dos como relevantes para cursar estudios superiores" (Chaín et al., 2003). Esto significa que, aun reconociendo que el buen desempeño depende de diversos factores, aquellos con mejores puntajes en estos exámenes generalmente tienen mayores probabilidades de realizar sus estudios en el sistema universitario, en tiempo y forma idóneos. Sin embargo, ¿cómo se podría definir la ponderación de dichos puntajes en un proceso de admisión a una Licenciatura en Música? A pesar de que se reconoce la importancia de los conocimientos y habilidades específicos del área musical en el proceso de selección, ¿son éstos más importantes que los conocimientos y habilidades intelectuales generales y los hábitos de estudio?, ¿cuánto más importantes son? Ésta es una de las problemáticas que podrían abordarse de manera fundamentada a través de proyectos de investigación sobre la eficiencia en los procesos de selección de candidatos de nuevo ingreso.

\section{Predictores académicos para estudiantes de música}

Los estudios sobre predictores del desempeño en música a nivel superior son menos comunes; en México no conocemos ningún reporte específico. Los reportes más antiguos encontrados en la literatura tienen relación con el efecto predictivo de la prueba general de aptitud musical, llamado Music Aptitude Profile [MAP]. Ésta fue desarrollada en Estados Unidos en la década de los años de 1960 para medir las aptitudes musicales de estudiantes de escuelas públicas (Gordon, 1967a, 1967b). La prueba está compuesta por tres módulos: 1) percepción de alturas -melodía y armonía-, 2) percepción de duraciones -tempo y métrica-, y 3 ) musicalidad -fraseo, balance y estilo-. Lee (1967) reportó el uso de esta prueba en estudiantes de música universitarios de nuevo ingreso ( $\mathrm{N}=57)$, encontrando una correlación significativa $(r=.35)$ entre los puntajes del MAP y el desempeño en las asignaturas de teoría musical -solfeo, armonía y entrenamiento auditivo-. Asimismo, el promedio de materias del área disciplinaria musical se correlacionó significativamente con los puntajes del MAP $(r=.38)$. No obstante, la correlación fue baja respecto al desempeño promedio que incluyó tanto materias disciplinarias como no disciplinarias, o del área académica general. En este caso, $r$ fue igual a .11 . De manera particular, el módulo de musicalidad alcanzó el coeficiente más alto $(r=.4)$ a un nivel de confianza de $1 \%$.

De manera similar, Schleuter y Schleuter (1978) encontraron un valor predictivo significativo de una adaptación del MAP para estudiantes universitarios $(\mathrm{N}=116)$, respecto al logro en habilidades auditivas, rítmicas y tonales, al final del 
primer semestre. A través de pruebas de regresión, encontraron que el MAP explicaba alrededor de un tercio de la variación del logro en los exámenes de habilidades auditivas. Los mejores predictores fueron los módulos melódico-armónico y rítmico del MAP, mientras que el de musicalidad contribuyó de manera marginal.

Más tarde, Schleuter (1993) integró los puntajes de la prueba estandarizada de ingreso (Scholastic Academic Test [SAT]) a la universidad y encontró que ésta no tenía un valor predictivo; mientras que la prueba específica de aptitudes musicales Advanced Measures of Music Audiation [AMMA] predecía el logro a primera vista y dictados en un grupo de 84 estudiantes de una licenciatura en música $(r=.45$ a $1 \%$ de confianza). Por el contrario, Arenson (1983) reportó una correlación significativa de los módulos de matemáticas y lenguaje del SAT con las calificaciones de las materias de teoría musical y solfeo al final del primer año ( $r$ entre .36 y .39); aunque las pruebas específicas de habilidades y aptitudes musicales fueron más determinantes ( $r$ entre .46 y .48).

Harrison (1990a, 1990b) y Harrison, Asmus y Serpe (1994) encontraron resultados análogos en el desempeño en entrenamiento auditivo y solfeo, en un grupo de 142 estudiantes que completaron el primer semestre de un curso de teoría musical. Lo innovador en el modelo hipotético fue la inclusión de cuatro variables predictivas del desempeño: la aptitud musical, medida a través del MAP; las habilidades académicas generales, medidas a través del sat; los años de experiencia musical; y la motivación hacia la música. El análisis multivariado resultó en una confirmación de las aptitudes musicales como el principal factor determinante del logro en solfeo y entrenamiento auditivo, seguido por las habilidades académicas generales -principalmente el módulo de matemáticas- y los años de experiencia. Extrañamente, la motivación, medida a través de autoreportes, no representó ninguna carga en el modelo.

Finalmente, en un estudio reciente, realizado en una universidad alemana, se exploró la relación entre los puntajes del examen de admisión específico para música -compuesto por una audición con el instrumento, una prueba de entrenamiento auditivo y otra de teoría musical-y las calificaciones de teoría musical, entrenamiento auditivo e instrumento principal, al final de la carrera. Lehmann (2014) (N= 93) reporta una correlación significativa, aunque moderada, entre las pruebas de ingreso y la calificación final de las materias de teoría y solfeo; mientras que la correlación del módulo de audición de la prueba de ingreso es débil con las demás variables, incluyendo la calificación de la materia de instrumento principal.

\section{Algunas reflexiones finales}

La presente revisión de la literatura marca una ruta de investigación que puede abordar las siguientes preguntas: ¿qué se puede predecir del desempeño académico de los estudiantes de la Licenciatura en Música de la UAA a partir de sus resultados en el proceso de admisión?, ¿cómo estas predicciones se deben reflejar en las ponderaciones que tienen el examen específico musical, el EXANI II y el promedio de bachillerato en el proceso de admisión?, ¿qué nos dice este análisis sobre la validez de dicho proceso?, ¿qué nos dice sobre los procesos de enseñanza y aprendizaje en la Licenciatura en Música?

Debido a los objetivos del proyecto de investigación, las variables predictivas del desempeño que guiaron la revisión de la literatura presentada fueron las variables académicas. Las más importantes son los exámenes estandarizados generales y específicos del campo musical, así como los resultados de etapas escolares previas; éstas son las mismas que se usan en el proceso de admisión de la Licenciatura en Música de la UAA. No obstante, la literatura tipifica otras susceptibles de ser exploradas. Harrison, Asmus y Serpe (1994) proponen un modelo que incluye, además, la motivación y la experiencia como ejecutante. Esta última, en particular, podría ser una variable importante en el contexto mexicano, si se consideran las áreas de oportunidad en la formación musical que presenta el sistema nacional escolarizado. El reto de incluir este tipo de variables es la manera en que se operacionalizan. En el estudio referido, la experiencia se mide en años como ejecutante, pero en nuestro contexto, y siguiendo nuestra preocupación central por las variables académicas, el constructo de experiencia podría ser una combinación entre años como ejecutante y tiempo en el que se recibió educación musical formal; pudiendo dividirse, esta última, en teórica -armonía, solfeo y entrenamiento auditivo-y práctica -ejecución musical-.

Un análisis del potencial predictivo en el proceso de admisión a una Licenciatura en Música puede realizarse a través de diversos modelos y técnicas. Los hallazgos expuestos de la literatura nos permiten tomar decisiones sobre posibles modelos hipotéticos que podremos usar en el caso de esta licenciatura de la UAA. La información institucional disponible es una limitante importante. Esperamos que en el presente artículo se haya expuesto información suficiente para que colegas de otros departamentos de música del país adapten nuestros planteamientos a sus necesidades específicas, y contribuyan a este campo del conocimiento, inexplorado en nuestro contexto nacional. Sólo a través de un estudio extenso, que coordine esfuerzos de diversas instituciones a nivel nacional, se podrán obtener datos válidos que permitan generalizaciones y sustenten la toma de decisiones. 
Fuentes de consulta

Albert, D. J. (2006). Socioeconomic Status and Instrumental Music: What Does the Research Say about the Relationship and Its Implications? Update: Applications of Research in Music Education, 25(1), 39-45. Recuperado de: https://doi.org/10.1177/87551233060250010 105.

Arenson, M. A. (1983). The Validity of Certain Entrance Tests as Predictors of Grades in Music Theory and Ear Training. Bulletin of the Council for Research in Music Education, (75), 33-39.

Arias, F., Chávez, A. y Muñoz, I. V. (2006). El aprovechamiento previo y la escuela de procedencia como predictores del aprovechamiento futuro: un caso. Enseñanza e Investigación en Psicología, 11(1).

Chaín, R., Cruz, N., Martínez, M. y Jácome, N. (2003). Examen de selección y trayectoria escolar. Revista de la Educación Superior, 32(125), 41-52.

Charles, Á. G. (2004). La relación entre el examen de admisión, el aprovechamiento académico y el Plan de Bachillerato 2000 en la Escuela de Bachilleres "Prof. Ladislao Farías Campos" de la Universidad Autónoma de Coahuila. Tesis de maestría Monclova, Coahuila: UAC.

Cortés, A. y Palomar, J. (2008). El proceso de admisión como predictor del rendimiento académico en la educación superior. Univérsitas Psychologica, 7(1), 1657-9267.

De Vries, W., Arenas, P., Muñoz, J. y Saldaña, I. (2011). ¿Desertores o decepcionados? Distintas causas para abandonar los estudios universitarios. Revista de la Educación Superior, XL(4) (160), 29-49.

Escudero, J. M. (1984). Orientaciones actuales en el currículum. Universidad de Murcia: Departamento de Currículum y Organización Escolar (inédito).

Garbanzo, G. M. (2007). Factores asociados al rendimiento académico en estudiantes universitarios, una reflexión desde la calidad de la educación superior pública. Revista educación, 31(1), 43-63.

González, M. y López E. (1985). Factores del rendimiento universitario. Revista Española de Pedagogía, No. 168, 497-519.

Gordon, E. (1967a). Implications for the Use of the "Musical Aptitude Profile" with College and University Freshman Music Students. Journal of Research in Music Education, 15(1), 32-40. Recuperado de: https://doi.org/10.2307/3344249.

Gordon, E. (1967b). The Musical Aptitude Profile. Music Educators Journal, 53(6), 52-54. Recuperado de: https://doi.org/10.2307/3390915.
Harrison, C. S. (1990a). Predicting Music Theory Grades: The Relative Efficiency of Academic Ability, Music Experience, and Musical Aptitude. Journal of Research in Music Education, 38(2), 124-137. Recuperado de: https://doi.org/10.2307/3344932.

Harrison, C. S. (1990b). Relationships between Grades in the Components of Freshman Music Theory and Selected Background Variables. Journal of Research in Music Education, 38(3), 175-186. Recuperado de: https://doi.org/10.2307/3345181.

Harrison, C. S., Asmus, E. P. y Serpe, R. T. (1994). Effects of Musical Aptitude, Academic Ability, Music Experience, and Motivation on Aural Skills. Journal of Research in Music Education, 42(2), 131-144. Recuperado de: https://doi.org/10.2307/3345497.

Lee, R. E. (1967). An Investigation of the Use of the Musical Aptitude Profile with College and University Freshman Music Students. Journal of Research in Music Education, 15(4), 278-288. Recuperado de: https://doi.org/10.2307/3343942.

Lehmann, A. C. (2014). Using Admission Assessments to Predict Final Grades in a College Music Program. Journal of Research in Music Education, 62(3), 245-258. Recuperado de: https://doi. org/10.1177/0022429414542654.

Morales, R., Barrera, A. y Garnett, E. (2005). Validez predictiva y concurrente del EXANI-II en la Universidad Autónoma del Estado de México. En $X$ Congreso Nacional de Investigación Educativa, 1-11.

Navarro, R. E. (2003). El Rendimiento Académico: Concepto, Investigación y Desarrollo. REICE Revista Electrónica Iberoamericana sobre Calidad, Eficacia y Cambio en Educación, 1(2). Recuperado de: https://doi.org/2152.

Schleuter, S. L. (1993). The Relationship of amma Scores to Sightsinging, Dictation, and SAT Scores of University Music Majors. Contributions to Music Education, (20), 57-63.

Schleuter, S. L. y Schleuter, L. J. (1978). A Predictive Study of an Experimental College Version of the "Musical Aptitude Profile" with Music Achievement of Non-Music Majors. Contributions to Music Education, (6), 2-8.

Touron, J. (1985). Predicción del rendimiento académico: Procedimientos, resultados e implicaciones. Revista Española de Pedagogía, 169-170, 473-495.

Wolf, A. y Kopiez, R. (2014). Do grades reflect the development of excellence in music students? The prognostic validity of entrance exams at universities of music. Musicae Scientiae, 18(2), 232-248. 